\title{
Marker infiammatori e rischio di fratture di bacino e vertebrali negli uomini: le fratture osteoporotiche negli uomini
}

\author{
S. Migliaccio ${ }^{1}$
}

Pubblicato online: 19 settembre 2016

(C) Springer International Publishing AG 2016

Commento a:

Inflammatory markers and the risk of hip and vertebral fractures in men: the osteoporotic fractures in men (MrOS).

J.A. Cauley, K.E. Barbour, S.L. Harrison,

Y.K. Cloonan, M.E. Danielson, K.E. Ensrud, H.A. Fink, E.S. Orwoll, R. Boudreau.

J Bone Miner Res (2016) doi:10.1002/jbmr.2905

L'osteoporosi maschile è una patologia altamente sottostimata, sotto-diagnosticata e sotto-trattata. Inoltre, se maggior peso è stato dato alla comprensione dei meccanismi coinvolti nel rischio di fratture nei soggetti di sesso femminile, poco è noto per quanto riguarda il genere maschile. La caratterizzazione dei fattori di rischio e dei meccanismi d'azione coinvolti nell'aumento del rischio di sviluppare fratture da fragilità è il primo passo per arrivare a una corretta diagnosi e intraprendere un corretto approccio di appropriatezza terapeutica.

Un lavoro appena pubblicato da Cauley e colleghi valuta il possibile coinvolgimento delle citochine proinfiammatorie nel determinismo del rischio di sviluppare fratture sia vertebrali che di femore in soggetti anziani di sesso maschile.

È noto come le citochine svolgano un ruolo importante nella regolazione del rimodellamento osseo, ma il loro rapporto con il rischio di fratture da fragilità negli uomini anziani non è mai stato valutato. In particolare, questo studio effettuato in soggetti facenti parte dell'ampia coorte di pazienti dello studio MrOs dimostra una correlazione di alcune

S. Migliaccio

silvia.migliaccio@uniroma4.it

1 Dipartimento di Scienze Motorie, Umane e della Salute, Unità di Endocrinologia, Università di Roma "Foro Italico", Roma, Italia specifiche citochine con il rischio di sviluppare fratture. Infatti, gli autori hanno valutato se soggetti di sesso maschile con livelli più elevati di marcatori pro-infiammatori avessero un rischio maggiore di fratture da fragilità. È stato effettuato uno studio caso-coorte e sono stati misurati marker infiammatori in un campione casuale di 961 uomini e in 120 uomini con fratture vertebrali cliniche, 117 con fratture di femore e 577 con fratture non-vertebrali. Il follow-up medio è stato di 6,13 anni (7,88 anni per le fratture vertebrali). Gli autori hanno valutato i livelli di IL-10, IL-6, PCR, TNF- $\alpha$, recettori solubili (SR) di IL-6 (IL-6SR) e TNF (TNF- $\alpha$ SR1 e TNF- $\alpha$ SR2).

Il rischio di fratture non vertebrali, di femore e fratture vertebrali cliniche è stato confrontato nei soggetti suddivisi in quartili (Q) sulla base dei livelli di marker infiammatori con analisi statistiche di modelli di rischio. L'analisi multivariata ha mostrato come gli uomini con le più alte concentrazioni (Q4) della citochina TNF- $\alpha$ e dei recettori specifici avessero un rischio 2,9 volte più elevato di fratture di femore e di fratture vertebrali cliniche rispetto agli uomini con valori più bassi (Q1). È interessante come $\mathrm{i}$ soggetti con più alti livelli di IL-10 avessero un rischio del $49 \%$ più basso di fratture vertebrali rispetto agli uomini nel quartile Q1. Tra gli uomini con più di 3 marcatori infiammatori nel più alto $\mathrm{Q}$, l'hazard ratio $(\mathrm{HR})$ per le fratture dell'anca era 2,03 (intervallo di confidenza al 95\% (CI): 1,11-3,71) e per fratture vertebrali 3,06 (95\% CI: 1,66-5,63). Non è risultata alcuna associazione tra infiammazione e tasso di perdita di densità minerale ossea dell'anca. In conclusione, i risultati ottenuti in questo studio dimostrano per la prima volta come l'infiammazione cronica possa svolgere un ruolo importante nell'eziologia delle fratture in soggetti anziani di sesso maschile, indicando l'importanza di controllare i valori di infiammazione cronica non solo nei soggetti di sesso femminile ma anche in quelli di sesso maschile. 